\title{
A Few Things We Do Not Know About Stars and Model Atmospheres
}

Robert L. Kurucz

Harvard-Smithsonian Center for Astrophysics. Cambridge, MA 02138, USA

April 20, 2001

\begin{abstract}
We list a few things that we do not understand about stars and that most people ignore. These are all hard problems. We can learn more cosmology by working on them to reduce the systematic errors they introduce than by trying to derive cosmological results that are highly uncertain.
\end{abstract}

\section{Optimism and Pessimism}

People sometimes complain that I am too pessimistic and that I criticize too much. In fact I am the most optimistic person. I believe that the human race is tremendously improvable and that humans can solve any problem. But the most important step in solving a problem is to realize that the problem exists. When I identify a problem I tell, or try to tell, the people who are capable of doing something about it. I also work on correcting the problem myself, if I am capable.

A pessimist does not believe that problems can be solved so does not question the present and does not search for errors. A pessimist acts so "optimistically" about the present that a pessimist prevents progress. Why worry about basic physics when everything is fine as it is?

The papers below are on my web site kurucz.harvard.edu. Some of them are also on the ASTRO-PH preprint server at Los Alamos.

A Few Things We Do Not Know About the Sun and F Stars and G Stars. I gave part of this talk at The Workshop on Nearby Stars at Ames two years ago. They wanted to know the state of the art in computing model atmospheres and spectra to determine whether they could see small abundance effects in the nearby stars or any spectral signature of planets. (I doubt it.)

Radiatively-Driven Cosmology. Most cosmologists never took a stellar atmospheres course and do not have experience with radiation. They do not realise the power of radiative acceleration compared to gravity.

A Correction to the pp Reaction. What if the pp reaction is a threebody reaction, two protons and an electron?

Vegan Astrophysics. This is a gedanken experiment to show the importance of basic physics. 


\section{We do not know how to make realistic model atmospheres; we do not understand convection}

Recently I have been preoccupied with convection because the model atmospheres are now good enough to show shortcomings in the convective treatment. Here I will outline what I have learned. I will mainly list the conclusions I have come to from examining individual convective models and from examining grids of convective models as a whole. Eighteen figures illustrating the points made here can be found in Kurucz (1996).

Every observation, measurement, model, and theory has seven characteristic numbers: resolution in space, in time, and in energy, and minimum and maximum energy. Many people never think about these resolutions. A low resolution physics cannot be used to study something in which the physical process of interest occurs at high resolution unless the high resolution effects average out when integrated over the resolution bandpasses.

What does the sun, or any convective atmosphere, actually look like? We do not really know yet. There is a very simplified three-dimensional radiation-hydrodynamics calculation discussed in the review by Chan, Nordlund, Steffen, and Stein (1991). It is consistent with the high spatial and temporal resolution observations shown in the review by Topka and Title (1991). Qualitatively, there is cellular convection with relatively slowly ascending, hot, broad, diverging flows that turn over and merge with their neighbors to form cold, rapidly descending, filamentary flows that diffuse at the bottom. The filling factor for the cold downward flowing elements is small. The structure changes with time. Nordlund and Dravins (1990) discuss four similar stellar models with many figures. Every one-dimensional mixing-length convective model is based on the assumption that the convective structure averages away so that the emergent radiation depends only a one-dimensional temperature distribution.

There is a solar flux atlas (Kurucz, Furenlid, Brault, and Testerman 1984) that Ingemar Furenlid caused to be produced because he wanted to work with the sun as a star for comparison to other stars. The atlas is pieced together from eight Fourier transform spectrograph scans, each of which was integrated for two hours, so the time resolution is two hours for a given scan. The $\mathrm{x}$ and $\mathrm{y}$ resolutions are the diameter of the sun. The $\mathrm{z}$ resolution (from the formation depths of features in the spectrum) is difficult to estimate. It depends on the signal-tonoise and the number of resolution elements. The first is greater than 3000 and the second is more than one million. It may be possible to find enough weak lines in the wings and shoulders of strong lines to 
map out relative positions to a few kilometers. Today I think it is to a few tens of kilometers. The resolving power is on the order of 522,000 . This is not really good enough for observations made through the atmosphere because it does not resolve the terrestrial lines that must be removed from the spectrum. (In the infrared there are many wavelength regions where the terrestrial absorption is too strong to remove.) The sun itself degrades its own flux spectrum by differential rotation and macroturbulent motions. The energy range of the atlas is from 300 to $1300 \mathrm{~nm}$, essentially the range where the sun radiates most of its energy.

This solar atlas is of higher quality than any stellar spectrum taken thus far but still needs considerable improvement. If we have difficulty interpreting these data, it can only be worse for other stars where the spectra are of lower quality by orders of magnitude.

To analyze this spectrum, or any other spectrum, we need a theory that works at a similar resolution or better. We use a plane parallel, one-dimensional theoretical or empirical model atmosphere that extends in $\mathrm{z}$ through the region where the lines and continuum are formed. The one-dimensional model atmosphere represents the space average of the convective structure over the whole stellar disk (taking account of the center-to-limb variation) and the time average over hours. It is usually possible to compute a model that matches the observed energy distribution around the flux maximum. However, to obtain the match it is necessary to adjust a number of free parameters: effective temperature, surface gravity, microturbulent velocity, and the mixing-length-to-scale-height-ratio in the one-dimensional convective treatment. The microturbulent velocity parameter also produces an adjustment to the line opacity to make up for missing lines. Since much of the spectrum is produced near the flux maximum, at depths in the atmosphere where the overall flux is produced, averaging should give good results. The parameters of the fitted model may not be those of the star, but the radiation field should be like that of the star. The sun is the only star where the effective temperature and gravity are accurately known. In computing the detailed spectrum, it is possible to adjust the line parameters to match many features, although not the centers of the strongest lines. These are affected by the chromosphere and by NLTE. Since very few lines have atomic data known accurately enough to constrain the model, a match does not necessarily mean that the model is correct.

From plots of the convective flux and velocity for grids of models I have identified three types of convection in stellar atmospheres:

- normal strong convection where the convection is continuous from the atmosphere down into the underlying envelope. Convection carries 
more than $90 \%$ of the flux. Stars with effective temperatures $6000 \mathrm{~K}$ and cooler are convective in this way as are stars on the main sequence up to $8000 \mathrm{~K}$. At higher temperature the convection carries less of the total flux and eventually disappears starting with the lowest gravity models. Intermediate gravities have intermediate behavior. Abundances have to be uniform through the atmosphere into the envelope. The highly convective models seem to be reasonable representations of real stars, except for the shortcomings cited below.

- atmospheric layer convection where, as convection weakens, the convection zone withdraws completely up from the envelope into the atmosphere. There is zero convection at the bottom of the atmosphere. Abundances in the atmosphere are decoupled from abundances in the envelope. For mixing-length models the convection zone is limited at the top by the Schwarzschild criterion to the vicinity of optical depth 1 or 2 . The convection zone is squashed into a thin layer. In a grid, this layer continues to carry significant convective flux for about $500 \mathrm{~K}$ in effective temperature beyond the strongly convective models. There is no common-sense way in which to have convective motions in a thin layer in an atmosphere. The solution is that the Schwarzschild criterion does not apply to convective atmospheres. The derivatives are defined only in one dimensional models. A real convective element has to decide what to do on the basis of local three-dimensional derivatives, not on means. These thin-layer-convective model atmospheres may not be very realistic.

- plume convection. Once the convective flux drops to the percent range, cellular convection is no longer viable. Either the star becomes completely radiative, or it becomes radiative with convective plumes that cover only a small fraction of the surface in space and time. Warm convective material rises and radiates. The star has rubeola. The plumes dissipate and the whole atmosphere relaxes downward. There are no downward flows. The convective model atmospheres are not very realistic except when the convection is so small as to have negligible effect, i.e. the model is radiative. The best approach may be simply to define a star with less than, say, $1 \%$ convection as radiative. The error will probably be less than using mixing-length model atmospheres.

Using a one-dimensional model atmosphere to represent a real convective atmosphere for any property that does not average in space and time to the one-dimensional model predictions produces systematic errors. The Planck function, the Boltzmann factor, and the Saha equation are functions that do not average between hot and cold convective elements. We can automatically conclude that one-dimensional convective models must predict the wrong value for any parameter that has strong exponential temperature dependence from these functions. 
Starting with the Planck function, ultraviolet photospheric flux in any convective star must be higher than predicted by a one-dimensional model (Bikmaev 1994). Then, by flux conservation, the flux redward of the flux maximum must be lower. It is fit by a model with lower effective temperature than that of the star. The following qualitative predictions result from the exponential falloff of the flux blueward of the flux maximum:

- the Balmer continuum in all convective stars is higher than predicted by a one-dimensional model;

- in G stars, including the sun, the discrepancy reaches up to about 400nm;

- all ultraviolet photoionization rates at photospheric depths are higher in real stars than computed from one-dimensional models;

- flux from a temperature minimum and a chromospheric temperature rise masks the increased photospheric flux in the ultraviolet;

- the spectrum predicted from a one-dimensional model for the exponential falloff region, and abundances derived therefrom, are systematically in error;

- limb-darkening predicted from a one-dimensional model for the exponential falloff region is systematically in error;

- convective stars produce slightly less infrared flux than do one-dimensional models.

The Boltzmann factor is extremely temperature sensitive for highly excited levels:

- the strong Boltzmann temperature dependence of the second level of hydrogen implies that the Balmer line wings are preferentially formed in the hotter convective elements. A one-dimensional model that matches Balmer line wings has a higher effective temperature than the real star;

- the same is true for all infrared hydrogen lines.

The Saha equation is safe only for the dominant species:

- neutral atoms for an element that is mostly ionized are the most dangerous because (in LTE) they are much more abundant in the cool convective elements. When Fe is mostly ionized the metallicity determination from Fe I can be systematically offset and can result in a systematic error in the assumed evolutionary track and age.

- in the sun convection may account for the remaining uncertainties with Fe I found by Blackwell, Lynas-Gray, and Smith (1995);

- the most striking case is the large systematic error in Li abundance determination in extreme Population II G subdwarfs. The abundance is determined from the $\mathrm{Li} \mathrm{I} \mathrm{D}$ lines which are formed at depths in the highly convective atmosphere where $\mathrm{Li}$ is $99.94 \%$ ionized (Kurucz 1995b); 
- molecules with high dissociation energies such as CO are also much more abundant in the cool convective elements. The CO fundamental line cores in the solar infrared are deeper than any one-dimensional model predicts (Ayres and Testerman 1981) because the cooler convective elements that exist only a short time have more $\mathrm{CO}$ than the mean model.

Given all these difficulties, how should we proceed? One-dimensional model atmospheres can never reproduce real convective atmospheres. The only practical procedure is to compute grids of model atmospheres, then to compute diagnostics for temperature, gravity, abundances, etc., and then to make tables of corrections. Say, for example, in using the $\mathrm{H} \alpha$ wings as a diagnostic of effective temperature in $\mathrm{G}$ stars, the models may predict effective temperatures that are $100 \mathrm{~K}$ too high. So if one uses an $\mathrm{H} \alpha$ temperature scale it has to be corrected by $100 \mathrm{~K}$ to give the true answer. Every temperature scale by any method has to be corrected in some way. Unfortunately, not only is this tedious, but it is very difficult or impossible because no standards exist. We do not know the energy distribution or the photospheric spectrum of a single star, even the sun. We do not know what spectrum corresponds to a given effective temperature, gravity, or abundances. The uncertainties in solar abundances are greater than 10\%, except for hydrogen, and solar abundances are the best known. It is crucial to obtain high resolution, high signal-to-noise observations of the bright stars.

\section{We do not consider the variation in microturbulent velocity}

Microturbulent velocity in the photosphere is just the convective motions. At the bottom of the atmosphere it is approximately the maximum convective velocity. At the temperature minimum it is zero or near zero because the convecting material does not rise that high. There is also microturbulent velocity in the chromosphere increasing outward from the temperature minimum that is produced by waves or other heating mechanisms. In the sun the empirically determined microturbulent velocity is about $0.5 \mathrm{~km} / \mathrm{s}$ at the temperature minimum and about $1.8 \mathrm{~km} / \mathrm{s}$ in the deepest layers we can see. In a solar model the maximum convective velocity is $2.3 \mathrm{~km} / \mathrm{s}$. The maximum convective velocity is about $0.25 \mathrm{~km} / \mathrm{s}$ in an $\mathrm{M}$ dwarf and increases up the main sequence. The convective velocity increases greatly as the

gravity decreases. I suggest that a good way to treat the behavior of microturbulent velocity in the models is to scale the solar empirical 
distribution as a function of Rosseland optical depth to the maximum convective velocity for each effective temperature and gravity.

Why does this matter? Microturbulent velocity increases line width and opacity and produces effects on an atmosphere like those from changing abundances. At present, models, fluxes, colors, spectra, etc are computed with constant microturbulent velocity within a model and from model to model. This introduces systematic errors within a model between high and low depths of formation, and between models with different effective temperatures, and between models with different gravity. Microturbulent velocity varies along an evolutionary track. If microturbulent velocity is produced by convection, microturbulent velocity is zero when there is no convection, and diffusion is possible.

By now I should have computed a model grid with varying microturbulent velocity but I am behind as usual.

\section{We do not understand spectroscopy; we do not have good spectra of the sun or any star}

Very few of the features called "lines" in a spectrum are single lines. Most features consist of blends of many lines from different atoms and molecules. All atomic lines except those of thorium have hyperfine or isotopic components, or both, and are asymmetric (Kurucz 1993). Low resolution, low-signal-to-noise spectra do not contain enough information in themselves to allow interpretation. Spectra cannot be properly interpreted without signal-to-noise and resolution high enough to give us all the information the star is broadcasting about itself. And then we need laboratory data and theoretical calculations as complete as possible. Once we understand high quality spectra we can look at other stars with lower resolution and signal-to-noise and have a chance to make sense of them.

\section{We do not have energy distributions for the sun or any star}

I get requests from people who want to know the solar irradiance spectrum, the spectrum above the atmosphere, that illuminates all solar system bodies. They want to interpret their space telescope observations or work on atmospheric chemistry, or whatever. I say, "Sorry, it has never been observed. NASA and ESA are not interested. I can give you my model predictions but you cannot trust them in detail, only in, say, one wavenumber bins." The situation is pathetic. 
I am reducing Brault's FTS solar flux and intensity spectra taken at Kitt Peak for .3 to $5 \mu \mathrm{m}$. I am trying to compute the telluric spectrum and ratio it out to determine the flux above the atmosphere but that will not work for regions of very strong absorption. Once that is done the residual flux spectra can be normalized to low resolution calibrations to determine the irradiance spectrum. The missing pieces will have to be filled in by computation. Spectra available in the ultraviolet are much lower resolution, much lower signal-to-noise, and are central intensity or limb intensity, not flux. The details of the available solar atlases can be found in two review papers, Kurucz (1991; 1995a).

\section{We do not know how to determine abundances; we do not know the abundances of the sun or any star}

One of the curiosities of astronomy is the quantity [Fe]. It is the logarithmic abundance of Fe in a galaxy, cluster, star, whatever, relative to the solar abundance of Fe. What makes it peculiar is that we do not yet know the solar abundance of Fe and our guesses change every year. The abundance has varied by a factor of ten since I was a student. Therefore $[\mathrm{Fe}]$ is meaningless unless the solar Fe abundance is also given so that $[\mathrm{Fe}]$ can be corrected to the current value of Fe.

For an example I use Grevesse and Sauval's (1999) solar Fe abundance determination. I am critical, but, regardless of my criticism, I still use their abundances. There are scores of other abundance analysis papers, including some bearing my name, that I could criticize the same way.

Grevesse and Sauval included 65 Fe I "lines" ranging in strength from 1.4 to $91.0 \mathrm{~m} \AA$ and $13 \mathrm{Fe}$ II "lines" ranging from 15.0 to $87.0 \mathrm{~m} \AA$. They found an abundance $\log \mathrm{Fe} / \mathrm{H}+12=7.50 \pm 0.05$.

Another curiosity of astronomy is that Grevesse and Sauval have decided a priori that the solar Fe abundance must equal the meteoritic abundance of 7.50 and that a determination is good if it produces that answer. If the solar abundance is not meteoritic, how could they ever determine it?

There are many "problems" in the analysis. First, almost all the errors are systematic, not statistical. Having many lines in no way

decreases the error. In fact, the use of a wide range of lines of varying strengths increases the systematic errors. Ideally a single weak line is all that is required to get an accurate abundance. Weak lines are relatively insensitive to the damping treatment, to microturbulent velocity, and to the model structure. The error is reduced simply by throwing out all lines greater than $30 \mathrm{~m} \AA$. That reduces the number of Fe I lines 
from 65 to 25 and of Fe II lines from 13 to 5 . As we discussed above, the microturbulent velocity varies with depth but Grevesse and Sauval assume that it is constant. This problem is minimized if all the lines are weak.

As we discussed above "lines" do not exist. The lines for which equivalent widths are given are all parts of blended features. As a minimum we have to look at the spectrum of each feature and determine how much of the feature in the "line" under investigation and how much is blending. Rigorously one should do spectrum synthesis of the whole feature. We have solar central intensity spectra and spectrum synthesis programs. For the sun we have the advantage of intensity spectra without rotational broadening. In the flux spectrum of the sun and of other stars there is more blending. The signal-to-noise of the spectra is several thousand and the continuum level can be determined to on the order of 0.1 per cent so the errors from the spectrum are small. With higher signal-to-noise more detail would be visible and the blending would be better understood. Most of the features cannot be computed well with the current line data. None of the features can be computed well without adjusting the line data. Even if the line data were perfect, the wavelengths would still have to be adjusted because of wavelength shifts from convective motions.

Fe has 4 isotopes. The isotopic splitting has not been determined for the lines in the abundance analysis. For weak lines it does not affect the total equivalent width but it does affect the perception of blends.

It is possible to have undetectable blends. There are many Fe I lines with the same wavelengths, including some in this analysis, and many lines of other elements. We hope that these blends are very weak. The systematic error always makes the observed line stronger than it is in reality so they produce an abundance overestimate.

There are systematic errors and random errors in the gf values. With a small number of weak lines on the linear part of the curve of growth it is easy to correct the abundances when the gf values are improved in the future.

We are left with 3 relatively safe lines of Fe I and 1 relatively safe line of Fe II. These have the least uncertainty in determining the blending by my estimation. Grevesse and Sauval found abundances of 7.455, 7.453, and 7.470 for the Fe I lines and 7.457 for the Fe II line. Thus from the same data the Fe abundances is 7.46 instead of 7.50. 


\section{We do not have good atomic and molecular data; one} half the lines in the solar spectrum are not identified

It is imperative that laboratory spectrum analyses be improved and extended, and that NASA and ESA pay for it. Some of the analyses currently in use date from the 1930s and produce line positions uncertain by 0.01 or $0.02 \AA$. New analyses with FTS spectra produce many more energy levels and one or two orders of magnitude better wavelengths. One analysis can affect thousands of features in a stellar spectrum. Also the new data are of such high quality that for some lines the hyperfine or isotopic splitting can be directly measured. Using Pickering (1996) and Pickering and Thorne (1996) I am now able to compute Co I hyperfine transitions and to reproduce the flag patterns and peculiar shapes of Co features in the solar spectrum. Using Litzen, Brault, and Thorne (1993) I am now able to compute the five isotopic transitions for $\mathrm{Ni}$ $\mathrm{I}$ and to reproduce the $\mathrm{Ni}$ features in the solar spectrum. These new analyses also serve as the basis for new semiempirical calculations than can predict the gf values and the lines that have not yet been observed in the lab but that matter in stars. I have begun to compute new line lists for all the elements and I will make them available on my web site, kurucz.harvard.edu.

\section{Cepheids have convective pulsation but the models do not; we do not have high quality spectra over phase for any Cepheid}

Cepheids are convective with velocities the same order of magnitude as the pulsation velocities. The sum of the velocities is supersonic and the difference is order zero. It is completely unphysical to try to compute the convection and the pulsation independently. Convective pulsation is a 3-dimensional radiation-hydrodynamics problem that must be solved as a whole.

If a hot Cepheid has a radiative phase, it becomes convective as it cools. The transition phase has space-time-random outward plumes that become supersonic. The surface is covered with spikes or bumps that cool by radiating toward the side.

All of this physics is displayed in the spectra of nearby Cepheids that are bright enough to be observed at $1 \mathrm{~km} / \mathrm{s}$ resolution and $\mathrm{S} / \mathrm{N}$ 3000. It would be perfectly feasible to make an atlas of such high resolution spectra every hour through the phases and then to read out the story, and also to use it to estimate boundary conditions for convective pulsation calculations. 


\section{We do not understand abundance evolution in early type stars}

This is a simplified, qualitative outline. Since there is no convection the atmosphere and upper layers mix very slowly. The bulk of the material of the star has approximately scaled solar abundances, $[\mathrm{Fe}] \geq$ 0 . When the star is formed the material in the atmosphere is the last to be accreted. It consists of dregs of the infall material that has been depleted of elements that are able to condense into grains. A young star has low metal abundances in the atmosphere and so appears to have $[\mathrm{Fe}]<<0$. As the star ages heavy elements with many lines are levitated into the atmosphere by radiative acceleration. Some elements, such as He, settle inward from gravity. The abundances become closer to solar, $[\mathrm{Fe}] \leq 0$. The star grows older and the abundances continue to increase in the atmosphere so that the star becomes a metallic line star with $[\mathrm{Fe}]>0$. If the star has strong magnetic spots, the abundances can be selectively enhanced by many orders of magnitude in the spots. The star is called "peculiar". A radiative wind selectively reduces abundances in the atmosphere because radiative acceleration affects some elements more than others. The only safe way to investigate early type stars is to obtain high quality spectra and spectrophotometry and to compute models and spectra for each star individually. Colors integrate away too many details. Astroseismology may be able to show abundance variation with depth.

From an evolutionary point of view, all main sequence early-type stars in our galaxy have slightly over solar abundances.

\section{Many early type stars are oblate fast rotators}

Early-type stars that are not in binaries are generally fast rotators. They are oblate because of the reduced gravity at the equator. The temperature can be several thousand degrees hotter at the poles than at the equator. Plane-parallel models like mine can be found that represent some average behavior but rigorously one must compute three dimensional rotating models. The real star has more ultraviolet flux from the poles and more infrared flux from the equator than the planeparallel models so the ionizing radiation field around an early-type star is prolate. It is probably not safe to use any unary early-type star as a photometric standard for calibrating theoretical photometry. 


\section{References}

Ayres, T.R. and Testerman, L. 1981, ApJ 245, 1124-1140.

Bikmaev, I. 1994, personal communication.

Blackwell, D.E., Lynas-Gray, A.E., and Smith, G. 1995, A\&A, 296, 217.

Chan, K.L., Nordlund, Å, Steffen, M., Stein, R.F. (1991) Solar Interior and Atmosphere, A.N. Cox, W.C. Livingston, and M. Matthews, eds. (Tucson: U. of Arizona Press) 223-274.

Grevesse, N. and Sauval, A.J. 1999, A\&A 347, 348-354.

Kurucz, R.L. 1991, in The Solar Interior and Atmosphere A.N. Cox, W.C. Livingston, and M. Matthews, eds., Tucson: U. of Arizona Press, 663-669.

Kurucz, R.L. 1993, Physica Scripta, T47, 110-117,

Kurucz, R.L. 1995a. in Laboratory and Astronomical High Resolution Spectra ASP Conf. Series 81, (eds. A.J. Sauval, R. Blomme, and N. Grevesse) 17-31.

Kurucz, R.L. 1995b. Ap.J.,452, 102-108.

Kurucz, R.L. 1996. in ASP Conf. Series 108, Model Atmospheres and Stellar Spectra (eds. S. Adelman, F. Kupka, and W.W. Weiss) 2-18.

Kurucz, R.L, Furenlid, I., Brault, J., and Testerman, L. 1984. Solar Flux Atlas from 296 to $1300 \mathrm{~nm}$ (Sunspot, N.M.: National Solar Observatory)

Litzen, U., Brault, J.W., and Thorne, A.P. 1993, Physica Scripta 47, 628-673.

Nordlund, A. and Dravins, D. 1990, A\&A 228, 155.

Pickering, J.C. 1996, ApJ Supp 107, 811-822.

Pickering, J.C. and Thorne, A.P. 1996, ApJ Supp 107, 761-809.

Topka, K.P. and Title, A.M. (1991) in Solar Interior and Atmosphere, A.N. Cox, W.C. Livingston, and M. Matthews, eds. (Tucson: U. of Arizona Press) 727-747. 